\title{
Performance Analysis of a Flexible Subsampling Receiver for Pulsed UWB Signals
}

\author{
Yves Vanderperren, Graduate Student Member, IEEE, Wim Dehaene, Senior Member, IEEE, \\ and Geert Leus, Senior Member, IEEE
}

\begin{abstract}
This paper presents a flexible digital receiver for pulsed Ultra-Wideband (UWB) communications which is sampling below Nyquist rate. This receiver can trade demodulation performance for sampling rate, i.e. power consumption. The bit error rate for pulse amplitude and pulse position modulations is evaluated in AWGN and typical UWB channels. The performance of several types of equalizer is compared, taking into account their implementation complexity. A suboptimal but implementation efficient Minimum Mean-Square Error (MMSE) equalizer which reaches performances similar to the ideal MMSE equalizer is proposed. The impact of imperfect knowledge of the propagation channel and signal-to-noise ratio, due to the limited number of training symbols, on the performance of the receiver is assessed. Finally, the receiver architecture and implementation cost are discussed. The proposed subsampling receiver provides an attractive alternative to classical architectures based on correlation with a template.
\end{abstract}

Index Terms-Ultra wideband (UWB), subsampling receiver, Nyquist.

\section{INTRODUCTION}

$\mathbf{T}$ HE design of pulsed UWB receivers presents unique challenges, due to the very large bandwidth of the transmitted signal. Analog UWB receivers, such as analog Rake (e.g., [1]) and Transmitted Reference (TR) systems [2], [3], avoid the need for high speed Analog-to-Digital Converters (ADC), but come at the cost of performance degradation and limited flexibility. Rake receivers cannot achieve the optimal bit error rate (BER) of the matched filter receiver since the number of fingers must be small for design complexity reasons [4], [5], whereas typical UWB channels present a high number of resolvable paths. In addition, multipathdependent pulse distortion [6] exacerbates the difficulty of generating a suitable template. TR systems avoid the need for local template generation by transmitting a modulated and unmodulated version of the pulse. However, this technique requires extremely wideband delay lines in the analog domain which are difficult to realize.

On the other hand, digital UWB receivers provide flexibility and benefit from CMOS technology scaling, but require an

Manuscript received October 18, 2007; revised October 16, 2008 and May 1, 2009; accepted May 7, 2009. The associate editor coordinating the review of this paper and approving it for publication was D. Dardari.

Y. Vanderperren and W. Dehaene are with the Department of Electrical Eng. (ESAT-MICAS), Katholieke Universiteit Leuven, 3001 Leuven, Belgium (e-mail: \{yves.vanderperren, wim.dehaene\} @esat.kuleuven.be).

G. Leus is with the Delft University of Technology, Faculty of Electrical Eng., Mathematics and Comp. Science (e-mail: g.j.t.leus@tudelft.nl).

This work was presented in part at the IEEE Conference on Communications (ICC), June 2006, Istanbul, Turkey.

Digital Object Identifier 10.1109/TWC.2009.080614
ADC sampling at Nyquist rate which is hardly realizable and highly power consuming, such as [7]. As the ADC power consumption of Flash ADCs, the standard solution for digital UWB architectures, scales linearly with the sampling rate and as a factor close to 4 with the bit width [8], several high speed 1-bit digital receiver architectures have been proposed, e.g. in [9], [10]. However, such receivers show limited robustness against interferers. Parallel multi-bit ADC architectures based on signal channelization in time [11] or frequency domain [12], [13] reach an aggregate sampling rate equivalent to Nyquist's criterion and support interference cancellation in the digital domain. This advantage over single 1-bit ADC architectures comes at the cost of increased area and power consumption, as each ADC typically consumes about 50-100 mW using state-of-the-art technology, e.g. [14][16]. Several Sigma-Delta ADCs in parallel could be used to improve the robustness against interference by virtue of oversampling. Still, these solutions require careful control of the circuit mismatches between the parallel branches.

Subsampling techniques provide an attractive alternative to traditional architectures. For example, a direct sampling approach based on a 2 GSamples/s ADC is proposed in [17]. However, it is only applicable for signals in the $3-5 \mathrm{GHz}$ band, due to the fixed relationship between the undersampling ratio and the selected band. In addition, the implementation of such a high speed ADC and the associated digital backend remains a challenging task. In this paper, we evaluate a subsampling receiver which recovers the position and the amplitude of the pulses by applying line spectrum estimation techniques in the frequency domain. This receiver provides the advantages of a digital implementation without the high power consumption and area penalty caused by a fast running or several low-speed ADCs. In addition, power consumption can be traded for demodulation quality simply by varying the sampling rate. As a result, this receiver provides a flexible alternative to traditional architectures.

This paper is structured as follows. In the next section, we describe the signal model and demodulation algorithm of the subsampling receiver. The performance for pulse position and amplitude modulations is discussed in Section III. The degradation caused by equalizer imperfections (noisy estimation of the propagation channel and signal-to-noise ratio) is verified in Section IV. The paper closes with an overview of the receiver architecture and complexity in Section V. 


\section{Application of Subsampling Techniques to Pulsed UWB Signals}

\section{A. Signal Model and Subsampled Pulse Detection Algorithms}

A transmitted pulsed UWB signal can be expressed as the convolution between a stream of Dirac impulses sent at frame rate $1 / T_{\mathrm{f}}$ and the transmitted pulse shape $p_{\mathrm{tx}}(t)$ :

$$
s(t)=p_{\mathrm{tx}}(t) * \sum_{n=-\infty}^{+\infty} \sum_{k=1}^{K} a_{n, k} \delta\left(t-n T_{\mathrm{f}}-t_{n, k}\right)
$$

where $a_{n, k} \in\{0, \pm A, \pm 3 A, \ldots\}$ and $t_{n, k} \in\{0, \Delta, 2 \Delta, \ldots\}$ are the data streams modulating $K$ pulse amplitudes and positions per period $T_{\mathrm{f}}$, respectively. For the sake of simplicity, we omit the Pseudo-Noise (PN) spreading code in (1) as the working principles of the subsampling receiver are independent of the spreading technique.

We first assume a channel impulse response $h(t)$ which does not modify the transmitted pulse shape, i.e. $h(t)=$ $\sum_{i=1}^{N_{\mathrm{p}}} \alpha_{i} \delta\left(t-\tau_{i}\right)$, where $N_{\mathrm{p}}$ is the total number of paths. The received pulsed UWB signal $r(t)$ can then be modeled as the convolution between a stream of Diracs with period $1 / T_{\mathrm{f}}$, the received pulse shape $p_{\mathrm{rx}}(t)$, and $h(t)$ :

$$
r(t)=p_{\mathrm{rx}}(t) * h(t) * \sum_{n=-\infty}^{+\infty} \sum_{k=1}^{K} a_{n, k} \delta\left(t-n T_{\mathrm{f}}-t_{n, k}\right)+n(t)
$$

where $n(t)$ is the received Additive White Gaussian Noise (AWGN). Assuming a pulse shape $p_{\mathrm{tx}}(t)$ with a spectrum centered at DC, the theory on sampling signals with finite rate of innovation [18] can be applied: the received signal $r(t)$ is filtered by an ideal low-pass filter with frequency response

$$
G_{\mathrm{lp}}(f)= \begin{cases}1 & |f| \leq M / T_{\mathrm{f}} \\ 0 & |f|>M / T_{\mathrm{f}}\end{cases}
$$

where $M \geq K$, and $M / T_{\mathrm{f}} \ll f_{\text {Nyquist. }}$. Let $g_{\mathrm{lp}}(t)=$ $M T_{\mathrm{f}}^{-1} \operatorname{sinc}\left(M T_{\mathrm{f}}^{-1} t\right)$ be the corresponding impulse response. The filtered signal $r_{\mathrm{lp}}(t)=r(t) * g_{\mathrm{lp}}(t)$ is sampled at a rate $f_{\mathrm{s}}=N_{\mathrm{f}} / T_{\mathrm{f}}$, with $N_{\mathrm{f}} \in \mathbb{N}$ and $N_{\mathrm{f}} \geq(2 M+1)$. The resulting samples $r_{\mathrm{lp}}[l]=r_{\mathrm{lp}}\left(l T_{\mathrm{s}}\right)$, with $T_{\mathrm{s}}=1 / f_{\mathrm{s}}$, are converted to the frequency domain by an FFT of size $N_{\mathrm{f}}$. Although the minimum sampling rate for a given half bandwidth $M / T_{\mathrm{f}}$ is $f_{\mathrm{s}, \min }=(2 M+1) / T_{\mathrm{f}}$, practical aspects such as efficient implementations of power of two FFTs suggest to adopt $f_{\mathrm{s}}=2^{\left\lceil\log _{2}(2 M+1)\right\rceil} / T_{\mathrm{f}}$ as sampling rate. In both cases, $N_{\mathrm{s}}=2 M+1$ frequency domain samples $r_{\mathrm{f}}^{(n)}[k]=$ $\sum_{m=0}^{N_{\mathrm{f}}-1} r_{\mathrm{lp}}\left[m+n N_{\mathrm{f}}\right] e^{-2 \pi i m k / N_{\mathrm{f}}}$, where $-M \leq k \leq M$, are available for each interval $I^{(n)}=\left[n T_{\mathrm{f}},(n+1) T_{\mathrm{f}}\right.$ [ of the received signal. Based on these samples, the construction of an annihilating filter is suggested in [18] in order to estimate the position of the pulses in each interval, under the assumption of noiseless conditions. In presence of noise, parametric Power Spectrum Density (PSD) estimation methods applied to $r_{\mathrm{f}}^{(n)}[k]$ provide a more robust solution [19]. In particular, line spectrum PSD estimation methods [20] can be used after deconvolving the received signal (2) by the pulse shape $p_{\text {rx }}(t)$. As further discussed in Section III-A, a particularly attractive property of these methods in the context of the subsampling approach is their ability to resolve pulses separated in time by less than $1 / f_{\mathrm{s}}$. This feature is the direct consequence of the ability of parametric methods for line spectra, when applied to time domain sinusoidal signals, to resolve spectral lines separated in frequency by less than $1 / T_{s}$. The deconvolution can be done by dividing $r_{\mathrm{f}}^{(n)}[k]$ by the pulse spectrum $p_{\mathrm{f}}[k]=\sum_{m=0}^{N_{\mathrm{f}}-1} p_{\mathrm{lp}}[m] e^{-2 \pi i m k / N_{\mathrm{f}}}$, with $-M \leq k \leq M$ and $p_{\mathrm{lp}}[m]=\left(p_{\mathrm{rx}} * g_{\mathrm{lp}}\right)\left(m T_{\mathrm{s}}\right)$. However, the number of paths which contribute significantly to the received energy is very high for typical UWB channels, requiring parametric estimation methods with unaffordable high order. It has been suggested in [21] to estimate a reduced set of principal components; yet, the order remains prohibitive ( $>10$ for Channel Model 1 in [22]) and affects the receiver complexity and the sampling rate. Moreover, it is assumed that the received pulse shape is known at the receiver, whereas it can actually differ significantly from the transmitted pulse shape and vary with the propagation paths. Pulse distortion is caused in particular by the transceiver antennas if these do not have a constant gain and linear phase frequency response [6]. Such distortion is hard to estimate independently from the channel effect.

A more realistic signal model, which takes into account the frequency selective distortion, is therefore given by

$$
r(t)=h_{\mathrm{c}}(t) * \sum_{n=-\infty}^{+\infty} \sum_{k=1}^{K} a_{n, k} \delta\left(t-n T_{\mathrm{f}}-t_{n, k}\right)+n(t)
$$

where $h_{\mathrm{c}}(t)=\sum_{i=1}^{N_{\mathrm{p}}} \alpha_{i} p_{i}\left(t-\tau_{i}\right)$ is the compound channel impulse response, which includes the distortion caused by the antennas and the dispersive behavior of the building materials in the propagation channel. As a result, the deconvolution by the pulse shape can not be applied. A combination of rational PSD estimation methods and a polynomial model for the frequency domain representation of $h_{\mathrm{c}}(t)$ is proposed in [23]. However, this approach still suffers from a high sampling rate caused by the required polynomial order. Instead, we suggest hereafter to deconvolve the received signal by the compound channel using an equalizer, and to apply a line spectrum PSD estimation method of minimal order, which provides more robust performance than parametric PSD estimation methods. In particular, we propose in this paper a simplified Minimum Mean-Square Error (MMSE) equalizer which avoids matrix operations without compromising the performance of the classical MMSE equalizer. This solution reduces the complexity of subsequent blocks: a line spectrum PSD estimation method of order $K$ only is required, instead of $K N_{\mathrm{p}}$.

\section{B. Basic Principles of the Line Spectrum Receiver}

Let $\mathbf{H}_{\mathrm{f}, \mathrm{c}}=\operatorname{diag}\left(\mathbf{h}_{\mathrm{f}, \mathrm{c}}\right)$ be defined as a diagonal matrix with the $N_{\mathrm{s}}$-point frequency domain representation of the filtered compound channel $\mathbf{h}_{\mathrm{f}, \mathrm{c}}=\left[h_{\mathrm{f}, \mathrm{c}}[-M] \ldots h_{\mathrm{f}, \mathrm{c}}[M]\right]^{T}$, with $h_{\mathrm{f}, \mathrm{c}}[k]=\sum_{m=0}^{N_{\mathrm{f}}-1} h_{\mathrm{lp}, \mathrm{c}}[m] e^{-2 \pi i m k / N_{\mathrm{f}}}$ for $-M \leq k \leq$ $M, h_{\mathrm{lp}, \mathrm{c}}[m]=\left(h_{\mathrm{c}} * g_{\mathrm{lp}}\right)\left(m T_{\mathrm{s}}\right)$. Under the assumption that $\max _{n, k}\left(t_{n, k}\right)+T_{\mathrm{d}}<T_{\mathrm{f}}$, where $T_{\mathrm{d}}$ is the delay spread of the filtered compound channel, the received signal is free of inter frame interference (IFI) and can be expressed in the frequency domain as

$$
\mathbf{r}_{\mathrm{f}}^{(n)}=T_{\mathrm{f}}^{-1} \mathbf{H}_{\mathrm{f}, \mathrm{c}} \mathbf{B}^{(n)} \mathbf{a}^{(n)}+\mathbf{n}^{(n)}
$$


where $\mathbf{r}_{\mathrm{f}}^{(n)}=\left[r_{\mathrm{f}}^{(n)}[-M] \ldots r_{\mathrm{f}}^{(n)}[M]\right]^{T}, \mathbf{n}^{(n)}$ corresponds to the filtered noise in the frequency domain affecting the $n^{\text {th }}$ symbol, $\mathbf{B}^{(n)}=\left[\begin{array}{lll}\mathbf{b}_{1}^{(n)} & \ldots & \mathbf{b}_{K}^{(n)}\end{array}\right], \mathbf{b}_{k}^{(n)}=\left[\begin{array}{ll}z_{n, k}^{-M} \ldots z_{n, k}^{M}\end{array}\right]^{T}$ with $z_{n, k}=e^{-2 \pi j t_{n, k} / T_{\mathrm{f}}}$, and $\mathbf{a}^{(n)}=\left[\begin{array}{lll}a_{n, 1} & \ldots & a_{n, K}\end{array}\right]^{T}$. We first assume $\mathbf{H}_{\mathrm{f}, \mathrm{c}}$ is perfectly known by the receiver. This assumption will be released in Section IV, where the impact of a limited number of training symbols is assessed. An equalization filter with frequency response $\mathbf{H}_{\mathrm{eq}}$ is constructed based on this training information, and the equalized signal computed: $\hat{\mathbf{s}}^{(n)}=\mathbf{H}_{\mathrm{eq}} \mathbf{r}_{\mathrm{f}}^{(n)}$. A line spectrum method of order $K$ is then applied to $\hat{\mathbf{s}}^{(n)}$ in order to estimate the positions $\left\{t_{n, i}\right\}_{i=1}^{K}$. We will assume the ESPRIT [24] method is applied, as it provides slightly more robustness against noise than other techniques, such as MUSIC [20], [25]. ESPRIT is based on a rotational invariance property of the signal subspace of the autocorrelation matrix $\mathbf{R}=N_{\mathrm{s}}^{-1} \mathbf{Y}^{H} \mathbf{Y}$, where $\mathbf{Y}$ is the data matrix associated to $\hat{\mathbf{s}}^{(n)}=\left[\hat{s}^{(n)}[-M] \ldots \hat{s}^{(n)}[M]\right]^{T}$ :

$$
\mathbf{Y}=\left[\begin{array}{ccc}
\hat{s}^{(n)}[-M+m-1] & \ldots & \hat{s}^{(n)}[-M] \\
\vdots & & \vdots \\
\hat{s}^{(n)}[M] & \ldots & \hat{s}^{(n)}[M-m+1]
\end{array}\right]
$$

The parameter $m$ in (6) can be freely chosen and allows trading the resolution of ESPRIT for robustness against noise. Let $\mathbf{V}_{\mathrm{s}}=\mathbf{V}\left[\mathbf{I}_{K} \mathbf{0}\right]^{T}$ be an $m \times K$ matrix with an orthonormal base of the signal subspace of $\mathbf{R}$, obtained from the Singular Value Decomposition (SVD) $\mathbf{Y}=\mathbf{U} \boldsymbol{\Sigma} \mathbf{V}^{H}$. By defining

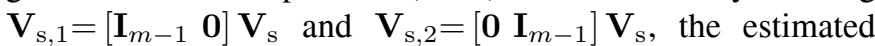
positions $\hat{\mathbf{t}}^{(n)}=\left[\begin{array}{lll}\hat{t}_{n, 1} & \ldots & \hat{t}_{n, K}\end{array}\right]^{T}$ are obtained as follows

$$
\hat{\mathbf{t}}^{(n)}=-\angle(\operatorname{eig}(\boldsymbol{\Phi}))
$$

with $\boldsymbol{\Phi}=\mathbf{V}_{\mathrm{s}, 1}^{+} \mathbf{V}_{\mathrm{s}, 2}$ and $\mathbf{V}_{\mathrm{s}, 1}^{+}$is the pseudoinverse of $\mathbf{V}_{\mathrm{s}, 1}$. The estimated amplitudes $\hat{\mathbf{a}}^{(n)}=\left[\begin{array}{lll}\hat{a}_{n, 1} & \ldots & \hat{a}_{n, K}\end{array}\right]^{T}$ are the least-squares solution of the system (5) after equalization:

$$
\hat{\mathbf{a}}^{(n)}=T_{\mathrm{f}} \hat{\mathbf{B}}^{(n)^{+}} \hat{\mathbf{s}}^{(n)}
$$

where $\hat{\mathbf{B}}^{(n)}$ is defined similarly to $\mathbf{B}^{(n)}$, using $\left\{\hat{t}_{n, i}\right\}_{i=1}^{K}$ instead of $\left\{t_{n, i}\right\}_{i=1}^{K}$.

The assumptions under which the signal model (5) is obtained are not critical. First, this model is appropriate for baseband pulses but can be extended to pulses in the 3.1-10.6 $\mathrm{GHz}$ frequency band by using an ideal bandpass filter

$$
G_{\mathrm{bp}}(f)= \begin{cases}1 & f_{\mathrm{c}}-M / T_{\mathrm{f}} \leq|f| \leq f_{\mathrm{c}}+M / T_{\mathrm{f}} \\ 0 & \text { otherwise }\end{cases}
$$

centered at a frequency $f_{c}$. As discussed in section $\mathrm{V}$, the selected band can be brought to baseband with a classical direct conversion architecture in order to avoid noise folding via aliasing. Second, non-ideal filters, such as Butterworth filters, can be used to allow for practical implementation of the receiver. Examples are given hereafter in Section III-C.

\section{Channel Equalization Alternatives}

We focus on linear equalizers working at symbol rate, such as Zero-Forcing (ZF) and Minimum Mean-Square Error (MMSE) equalizers. Fractionally spaced equalizers exacerbate the sampling rate issue and are not suitable for subsampling digital UWB receivers.
1) $Z F$ : In this case, $\mathbf{H}_{\mathrm{eq}}=\mathbf{H}_{\mathrm{f}, \mathrm{c}}^{-1}$ and the deconvolution by $h_{\mathrm{c}}(t)$ is implemented as a division in the frequency domain. This straightforward solution requires only the inversion of $N_{\mathrm{s}}$ complex numbers.

2) Optimal MMSE: The MMSE equalizer minimizes $\mathcal{J}=\mathrm{E}\left\{\left\|\mathbf{s}^{(n)}-\hat{\mathbf{s}}^{(n)}\right\|^{2}\right\}$, where $\mathbf{s}^{(n)}=\mathbf{B}^{(n)} \mathbf{a}^{(n)}$. Solving for $\partial \mathcal{J} / \partial \mathbf{H}_{\mathrm{eq}}=0$, the expression of the MMSE is given by

$$
\mathbf{H}_{\mathrm{eq}}=\mathbf{R}_{\mathrm{s}} \mathbf{H}_{\mathrm{f}, \mathrm{c}}^{H}\left(\mathbf{H}_{\mathrm{f}, \mathrm{c}} \mathbf{R}_{\mathrm{s}} \mathbf{H}_{\mathrm{f}, \mathrm{c}}^{H}+\mathbf{R}_{\mathrm{n}}\right)^{-1}
$$

where $\mathbf{R}_{\mathrm{S}}=\mathrm{E}\left\{\mathbf{s}^{(n)} \mathbf{S}^{(n)^{H}}\right\}$ and $\mathbf{R}_{\mathrm{n}}=\mathrm{E}\left\{\mathbf{n}^{(n)} \mathbf{n}^{(n)^{H}}\right\}$ are the data and noise covariance matrices in the frequency domain. The training of this equalizer requires the inversion of an $N_{\mathrm{s}} \times N_{\mathrm{s}}$ complex matrix. The equalization of each data symbol $\mathbf{r}_{\mathrm{f}}^{(n)}$ by $\mathbf{H}_{\mathrm{eq}}$ involves a complex matrix multiplication which requires $2 N_{\mathrm{s}}^{2}$ operations.

3) Suboptimal MMSE: Provided that the pulse shape has a flat spectrum in the band selected by the receiver, we can relax the assumption of colored noise and signal covariance matrices. By approximating $\mathbf{R}_{\mathrm{s}}$ and $\mathbf{R}_{\mathrm{n}}$ with their diagonal, (10) requires only the inversion of $N_{\mathrm{s}}$ numbers, since $\mathbf{H}_{\mathrm{f}, \mathrm{c}}$ is already diagonal. The estimation of the approximated noise covariance matrix $\mathbf{R}_{\mathrm{n}, \text { diag }}=\sigma_{\mathrm{n}}^{2} \mathbf{I}_{N_{\mathrm{s}}}$ requires only the estimation of the noise power at the equalizer input, which can be done during training. The approximated signal covariance matrix $\mathbf{R}_{\mathrm{s}, \text { diag }}=\sigma_{\mathrm{s}}^{2} \mathbf{I}_{N_{\mathrm{s}}}$ is known at the receiver as the Voltage Gain Amplifier (VGA) sets the signal power at a known reference level dictated by the Automatic Gain Control (AGC), irrespective of the received power at the antenna. Defining $S N R=\sigma_{\mathrm{s}}^{2} / \sigma_{\mathrm{n}}^{2}$, the suboptimal MMSE equalizer can be expressed as

$$
\mathbf{H}_{\mathrm{eq}}=\mathbf{H}_{\mathrm{f}, \mathrm{c}}^{H}\left(\mathbf{H}_{\mathrm{f}, \mathrm{c}} \mathbf{H}_{\mathrm{f}, \mathrm{c}}^{H}+S N R^{-1} \mathbf{I}_{N_{\mathrm{s}}}\right)^{-1}
$$

and only involves scalar operations with a complexity of $\mathcal{O}\left(N_{\mathrm{s}}\right)$. Similarly, the equalization of each data symbol requires $N_{\mathrm{s}}$ multiplications, like the $\mathrm{ZF}$ equalizer. As a result, this suboptimal MMSE equalizer avoids costly matrix inversions and multiplications.

\section{Performance of the Subsampling Receiver}

Monte-Carlo simulations have been carried out in order to assess the performance of the line spectrum subsampling receiver with an AWGN channel and in multipath conditions [22]. Given a desired $\left(E_{\mathrm{b}} / N_{0}\right)_{\text {des }}$, the AWGN noise power added in the discrete time simulation to the transmitted signal is

$$
\sigma_{\mathrm{n}, \mathrm{in}}^{2}=\frac{\sigma_{\mathrm{s}, \mathrm{in}}^{2}}{\left(E_{\mathrm{b}} / N_{0}\right)_{\mathrm{des}}} \cdot \frac{W}{K / T_{\mathrm{f}}}
$$

where $\sigma_{\mathrm{s}, \text { in }}^{2}$ is the average received signal power and $W$ the model bandwidth. This allows for a fair comparison of the receiver performance when using different pulse types and filter bandwidths. Although the simulation results presented in this paper have been obtained with the second derivative of a Gaussian monocycle occupying the $3.1-10.6 \mathrm{GHz}$ band, no significant difference was observed with other pulses.

Different receiver bandwidths and sampling rates have been simulated, and a single pulse per period is assumed $(K=1)$. The pulse repetition period is high enough $\left(T_{\mathrm{f}}=51.2 \mathrm{~ns}\right)$ to 


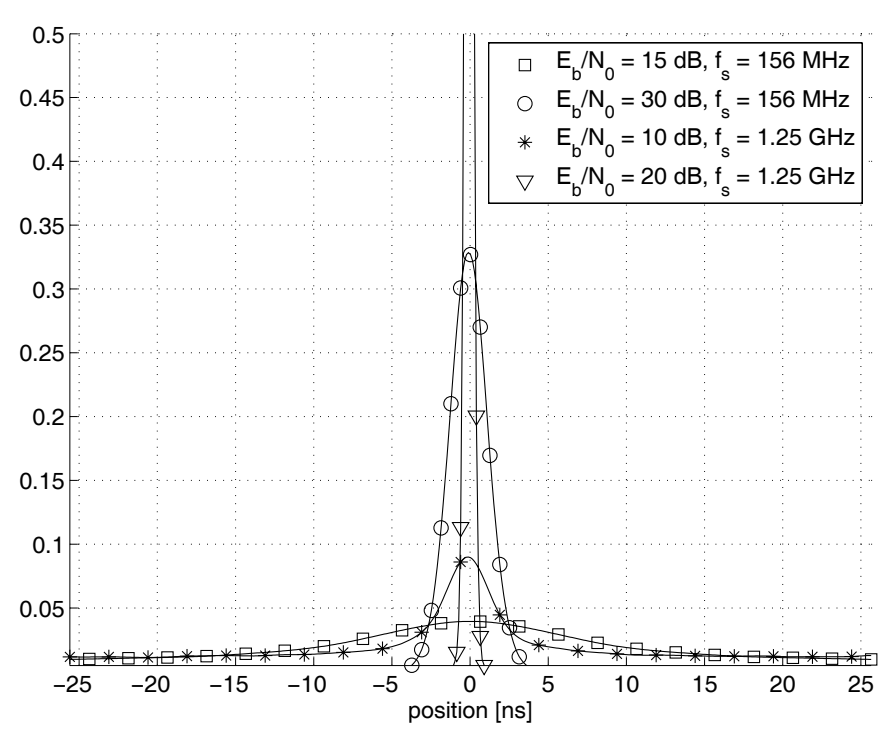

Fig. 1. Examples of PDF of the estimated pulse position.

avoid IFI. The central frequency $f_{\mathrm{c}}$ of the receiver bandpass filter is chosen as the maximum of the pulse PSD.

\section{A. Distribution of the Estimated Position and BER for PPM}

Figure 1 shows typical examples of the distribution of the position estimated by ESPRIT for different levels of $E_{\mathrm{b}} / N_{0}$ and sampling rates in an AWGN channel. At low $E_{\mathrm{b}} / N_{0}$ and sampling rate, the error on the estimated pulse position is in the order of magnitude of the pulse repetition period. This extreme situation is not suitable for reliable communication. At higher $E_{\mathrm{b}} / N_{0}$ or sampling rates, the pulse position can be very accurately estimated with a maximum error below 1 ns at $E_{\mathrm{b}} / N_{0}>20 \mathrm{~dB}$ and $f_{\mathrm{s}}=1.25 \mathrm{GHz}$. Indeed, a remarkable property [26] of line spectrum methods, applied in this context in the frequency domain instead of the time domain, is the dependency in $\mathcal{O}\left(N_{\mathrm{s}}^{-3}\right)$ of the variance of the estimated pulse position $\sigma_{\mathrm{t}}^{2}=E\left[\left(\hat{t}_{n, 1}-t_{n, 1}\right)^{2}\right]$ with the number of available samples, i.e. with the receiver bandwidth and the ADC sampling rate:

$$
\sigma_{\mathrm{t}}^{2} \approx \frac{\sigma_{\mathrm{n}}^{2} T_{\mathrm{f}}^{2}}{4 \pi^{2} m\left(N_{\mathrm{s}}-m\right)^{2} \sigma_{\mathrm{s}}^{2}}
$$

As a result, the performance improves smoothly and rapidly with increasing bandwidths. Conversely, the receiver degrades gracefully with lower sampling rates instead of suffering from a severe performance penalty due to sub-Nyquist sampling. Furthermore, the dependency of $\sigma_{\mathrm{t}}^{2}$ in $\mathcal{O}\left(N_{\mathrm{s}}^{-3}\right)$ instead of $\mathcal{O}\left(N_{\mathrm{s}}^{-2}\right)$ allows pulses separated by less than $1 / f_{\mathrm{s}}$ to be distinguished. This property is equivalent to the ability of these methods, applied traditionally in the time domain to sinusoidal signals, to estimate frequencies separated by less than $f_{\mathrm{s}} / N$, where $N$ is the number of samples. Finally, it should be observed the receiver can reach a performance close to the optimum. Indeed, the lowest estimation error for a given pulse period and sampling rate is achieved for $m=N_{\mathrm{s}} / 3$, in which case

$$
\sigma_{\mathrm{t}}^{2} \approx \frac{27 \sigma_{\mathrm{n}}^{2} T_{\mathrm{f}}^{2}}{16 \pi^{2} N_{\mathrm{s}}^{3} \sigma_{\mathrm{s}}^{2}}
$$

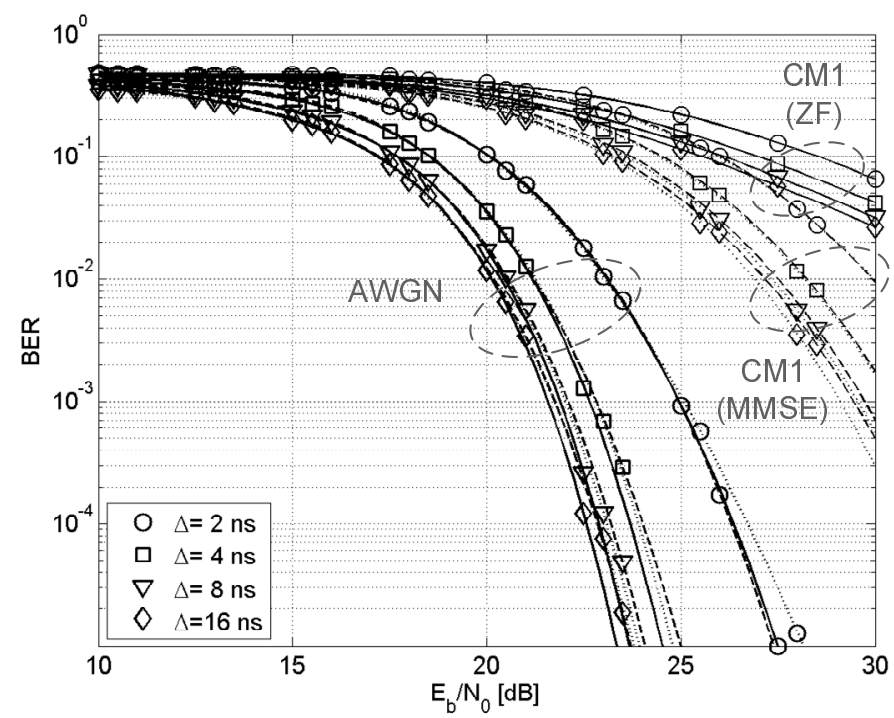

Fig. 2. BER PPM, $f_{\mathrm{s}}=625 \mathrm{MHz}$, with ZF (solid), MMSE (dotted), and simplified MMSE (dashed) equalizers.

which is very close to the Cramer-Rao bound

$$
\sigma_{\mathrm{t}, \mathrm{CRB}}^{2} \approx \frac{3 \sigma_{\mathrm{n}}^{2} T_{\mathrm{f}}^{2}}{2 \pi^{2} N_{\mathrm{s}}^{3} \sigma_{\mathrm{s}}^{2}}
$$

As a result, the performance degradation of the subsampling receiver with respect to the ideal receiver, based on perfect matched filtering, is essentially caused by the limited fraction of signal energy which is captured by the bandpass filter in the receiver front-end. A subsampling receiver based on line spectrum estimation techniques provides therefore a particularly attractive solution compared to conventional receivers for pulsed UWB.

The Probability Density Function (PDF) of the estimated position depends on $E_{\mathrm{b}} / N_{0}$ and the number of samples. At low $E_{\mathrm{b}} / N_{0}$ and sampling rates, it presents a heavy tail which causes the rejection of the null hypothesis of a normal distribution at 5\% confidence level by a KolmogorovSmirnov (K-S) test. Indeed, it is known that the estimates of sinusoidal frequencies using subspace rotation techniques are not Gaussian distributed [25], although the distribution is asymptotically normal [27]. The number of available samples from subsampled UWB signals, with a pulse repetition rate and a sampling rate in the order of magnitude of tens to hundreds of $\mathrm{MHz}$, is too small compared to the asymptotic case. However, the K-S test becomes positive for $f_{\mathrm{s}}>500 \mathrm{MHz}$ and $E_{\mathrm{b}} / N_{0}>25 \mathrm{~dB}$.

Figs. 2 and 3 illustrate the BER curves for PPM for various sampling rates as a function of the modulation index $\Delta$. The MMSE and ZF equalizers present the same performance in an AWGN channel, since only the pulse PSD, which is almost flat in the considered band, is equalized. Since the estimated pulse position follows asymptotically a Gaussian distribution in AWGN channel conditions, we can approximate the BER expression as

$$
P_{B} \approx Q\left(\sqrt{\frac{\eta E_{b}}{N_{0}}}\right)
$$

where $\eta$ is the fraction of signal energy captured by the 


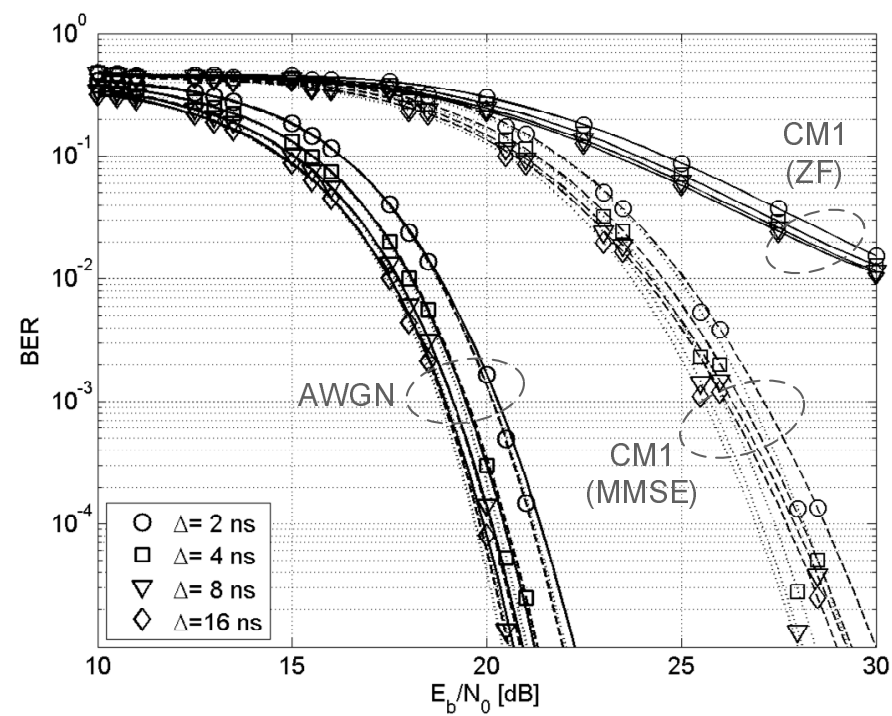

Fig. 3. BER PPM, $f_{\mathrm{s}}=1.25 \mathrm{GHz}$, with ZF (solid), MMSE (dotted), and simplified MMSE (dashed) equalizers.

bandpass filter. This fraction is proportional to the bandwidth of the filter and can be approximated as $\eta \approx\left(N_{\mathrm{s}} / T_{\mathrm{f}}\right) / B$, where $B$ is the signal bandwidth, $B \approx(10.6-3.1)=7.5 \mathrm{GHz}$. Following these approximated expressions, the degradation at $f_{\mathrm{s}}=1.25 \mathrm{GHz}$, for example, with respect to an ideal matched filter receiver is in the order of magnitude of $8 \mathrm{~dB}$ and is in agreement with the simulation results for an AWGN channel in Fig. 3.

When considering realistic multipath conditions such as CM1, which present numerous amplitude dips in their frequency response, the ZF BER may still be close to the MMSE for particular channel realizations. However, the MMSE outperforms the ZF equalizer when the results are averaged over a high number (100) of realizations. The noise power term in (10) and (11) prevents noise enhancement at these locations, as confirmed by the increasing performance improvement of the MMSE vs. the ZF equalizer for larger receiver bandwidths and sampling rates. Interestingly, the suboptimal MMSE based on diagonal covariance matrices does not introduce any significant BER penalty for the sampling rates which allow for reliable communication, i.e. $M_{\min } \approx 8$ or $f_{\mathrm{s}, \min } \approx 16 / T_{\mathrm{f}}$. Indeed, the receiver bandwidth is then sufficiently high, compared to the pulse repetition rate, to validate the approximation of the signal and noise covariance matrices by their diagonal.

\section{B. Distribution of the Estimated Amplitude and BER for PAM}

Similarly to PPM, the estimated amplitude follows a normal distribution at high sampling rate. The BER curves for PAM modulation and various sampling rates (Fig. 4) give rise to conclusions similar to PPM. The superior results of the MMSE vs. the ZF equalizer for increasing bandwidth are visible in figure 4 for the curves averaged over 100 different CM1 realizations.

The conclusions of these results for PAM and PPM are that 1) the MMSE equalizer provides a clear performance advantage compared to a ZF solution, and 2) the simplified MMSE provides a BER similar to the optimal MMSE but
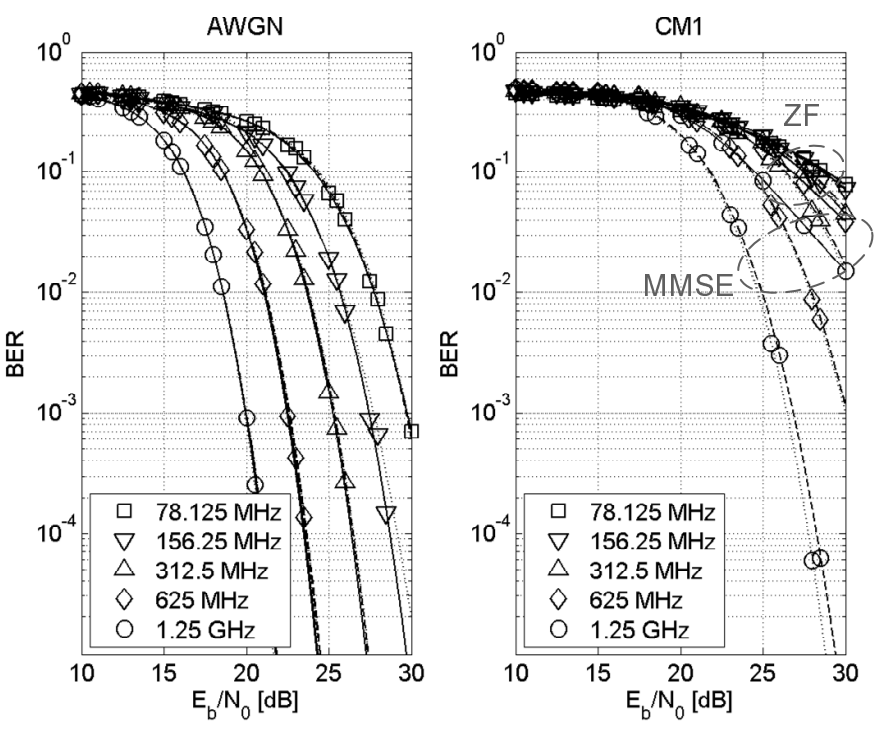

Fig. 4. BER PAM, with ZF (solid), MMSE (dotted), and simplified MMSE (dashed) equalizers.

at much lower complexity, as it avoids inverting an $N_{\mathrm{s}} \times N_{\mathrm{s}}$ matrix.

\section{Application of Realistic Filters}

Ideal filters were assumed so far in order to evaluate the performance of the receiver independently of implementation aspects. In presence of a realistic filter with impulse response $g(t)$, the signal model (5) can be expressed as

$$
\mathbf{r}_{\mathrm{f}}^{(n)}=T_{\mathrm{f}}^{-1} \mathbf{G}_{\mathrm{f}} \mathbf{H}_{\mathrm{f}, \mathrm{c}} \mathbf{B}^{(n)} \mathbf{a}^{(n)}+\mathbf{n}^{(n)}
$$

where $\mathbf{G}_{\mathrm{f}}=\operatorname{diag}\left(\mathbf{g}_{\mathrm{f}}\right)$ represents the sampled frequency domain response of the filter, where $\mathbf{g}_{\mathrm{f}}=\left[g_{\mathrm{f}}[-M] \ldots g_{\mathrm{f}}[M]\right]^{T}$, and $g_{\mathrm{f}}[k]=\sum_{m=0}^{N_{\mathrm{f}}-1} g\left(m T_{\mathrm{s}}\right) e^{-2 \pi i m k / N_{\mathrm{f}}}$ for $-M \leq k \leq M$. The working principles of the receiver remain applicable provided that $f_{\mathrm{s}} \geq 2 f_{0}$, where $f_{0}$ is the cut-off frequency of the filter. This condition guarantees that the amount of aliasing due to the transition band and the limited rejection in the stopband remains negligible.

For a given $f_{\mathrm{s}}$, the parameter $M$, which specifies the number of frequency domain samples effectively used within the $N_{\mathrm{f}}$ available samples, can be freely chosen. By defining $\tilde{\mathbf{H}}_{\mathrm{f}, \mathrm{c}}=\mathbf{G}_{\mathrm{f}} \mathbf{H}_{\mathrm{f}, \mathrm{c}}$ and replacing $\mathbf{H}_{\mathrm{f}, \mathrm{c}}$ by $\tilde{\mathbf{H}}_{\mathrm{f}, \mathrm{c}}$ in (11), the equalizer will indeed attempt to compensate for the attenuation of the samples $r_{\mathrm{f}}^{(n)}[k], f_{0} T_{\mathrm{f}} \leq|k| \leq M$, which lie outside of the passband. In practice, the best performance is obtained when $M \approx f_{0} T_{\mathrm{f}}$, so that all selected samples lie in the passband.

As an example, Fig. 5 illustrates the BER curves for PPM modulation with different filters in AWGN conditions. In all cases, $f_{0}=f_{\mathrm{s}} / 2$ and $M=f_{0} T_{\mathrm{f}}$. The simplified MMSE equalizer (11) is assumed. The filter response is therefore not taken into account in the equalizer, in order to observe the impact of the attenuated frequency domain samples which are located close to the cut-off frequency. As it can be seen from Figs. 2, 3 and 5, the degradation is limited to $2-4 \mathrm{~dB}$. This scenario corresponds to a worst case situation. In practice, the compound channel impulse response seen by the equalizer 


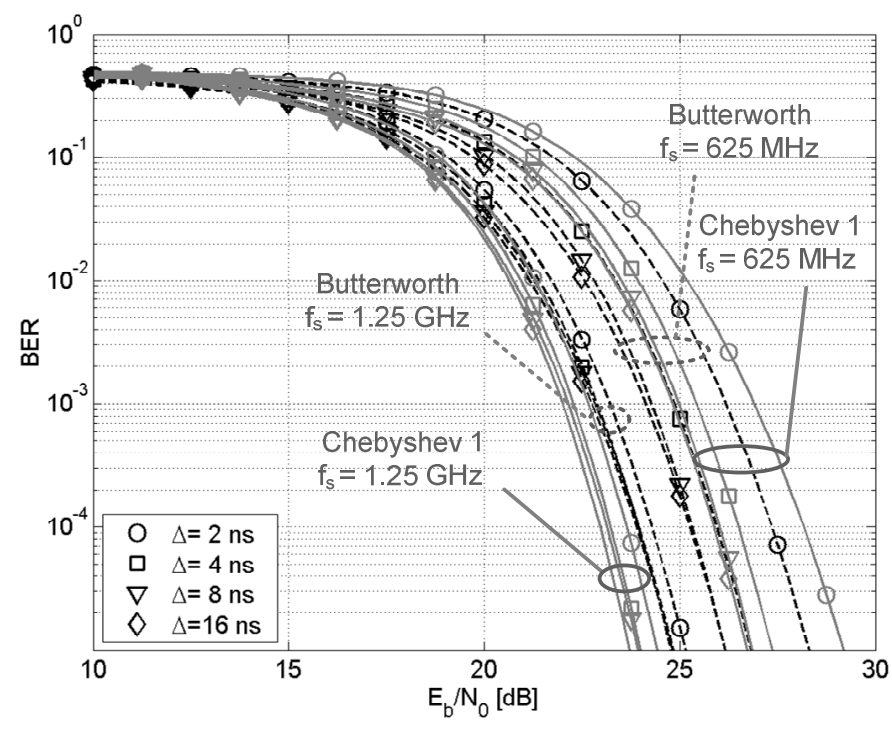

Fig. 5. Examples of BER for $4^{\text {th }}$ order Butterworth and $2^{\text {nd }}$ order Chebyshev Type I filters. The ripple factor of the Chebyshev filter is $1 \mathrm{~dB}$.

during training will include the filter response, and the same performance as in Figs. 2-3 will be achieved.

\section{IMPACT OF IMPERFECT EQUALIZATION}

\section{A. Noisy Estimation of the Propagation Channel}

In this section, we release the assumption of perfect knowledge of the propagation channel. Instead, the receiver is assumed to obtain $\hat{\mathbf{h}}_{\mathrm{f}, \mathrm{c}}$, the minimum variance unbiased estimation of $\mathbf{h}_{\mathrm{f}, \mathrm{c}}$, from a noisy sequence of $N_{\mathrm{pch}}$ training symbols $\mathbf{r}_{\mathrm{f}}^{(j)}, 0 \leq j \leq N_{\mathrm{pch}}-1: \hat{\mathbf{h}}_{\mathrm{f}, \mathrm{c}}=N_{\mathrm{pch}}^{-1} \sum_{j=0}^{N_{\mathrm{pch}}-1} \mathbf{r}_{\mathrm{f}}^{(j)}$. The noisy suboptimal MMSE equalization filter with frequency response $\hat{\mathbf{H}}_{\text {eq }}$ is then constructed:

$$
\hat{\mathbf{H}}_{\mathrm{eq}}=\hat{\mathbf{H}}_{\mathrm{f}, \mathrm{c}}^{H}\left(\hat{\mathbf{H}}_{\mathrm{f}, \mathrm{c}} \hat{\mathbf{H}}_{\mathrm{f}, \mathrm{c}}^{H}+\sigma_{\mathrm{n}}^{2} / \sigma_{\mathrm{s}}^{2} \mathbf{I}_{N_{\mathrm{s}}}\right)^{-1}
$$

where $\hat{\mathbf{H}}_{\mathrm{f}, \mathrm{c}}=\operatorname{diag}\left(\hat{\mathbf{h}}_{\mathrm{f}, \mathrm{c}}\right)$.

1) Degradation in Terms of Signal-to-Noise Ratio: The degradation in terms of SNR caused by the noisy channel estimation can be estimated as follows. Let $\mathbf{h}_{\mathrm{eq}}$ and $\hat{\mathbf{h}}_{\mathrm{eq}}$ be defined as

$$
\begin{aligned}
& \mathbf{h}_{\text {eq }}=\left[h_{\text {eq }}[-M] \ldots h_{\text {eq }}[M]\right]^{T}=\operatorname{diag}\left(\mathbf{H}_{\text {eq }}\right) \\
& \hat{\mathbf{h}}_{\text {eq }}=\left[\hat{h}_{\text {eq }}[-M] \ldots \hat{h}_{\text {eq }}[M]\right]^{T}=\operatorname{diag}\left(\hat{\mathbf{H}}_{\text {eq }}\right)
\end{aligned}
$$

The equalized symbol $\hat{\mathbf{s}}=[\hat{s}[-M] \ldots \hat{s}[M]]^{T}$, where we omit the symbol index $n$ for readability as we focus on the demodulation of a single symbol, can be expressed as

$$
\begin{aligned}
\hat{s}[k] & =\left(h_{\mathrm{f}, \mathrm{c}}[k] s[k]+n[k]\right) \hat{h}_{\mathrm{eq}}[k] \\
& =\left(h_{\mathrm{f}, \mathrm{c}}[k] s[k]+n[k]\right)\left(h_{\mathrm{eq}}[k]+n_{\mathrm{h}}[k]\right)
\end{aligned}
$$

where $n_{\mathrm{h}}[k]$ is the noise affecting $h_{\mathrm{eq}}[k]$ and $-M \leq k \leq M$. The SNR at the input of the equalizer is given by

$$
(S N R)_{\text {in }}=10 \log _{10}\left(E\left[\left|h_{\mathrm{f}, \mathrm{c}} s\right|^{2}\right] / E\left[|n|^{2}\right]\right)
$$

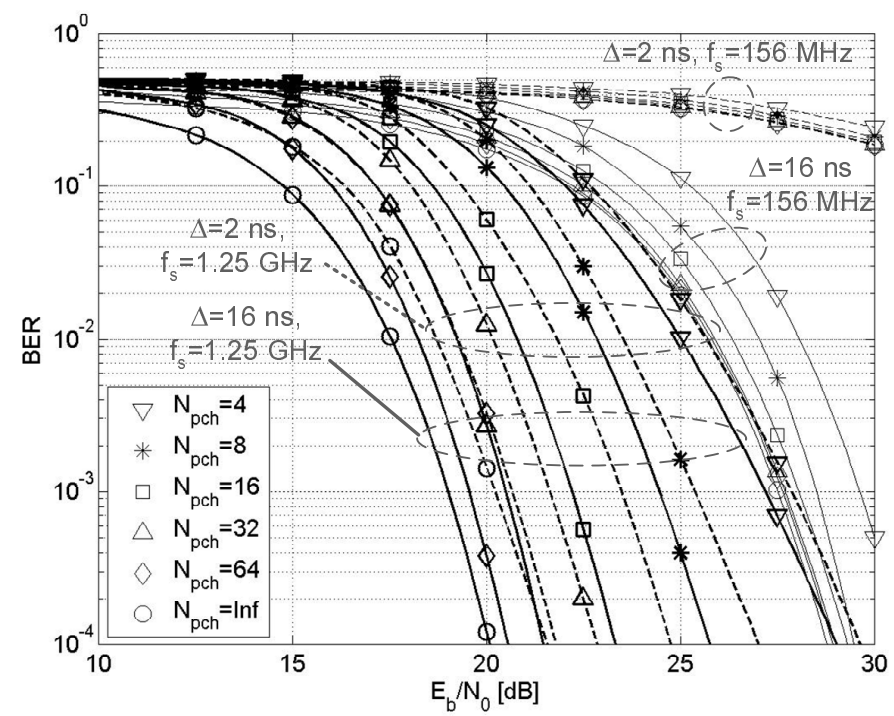

Fig. 6. BER for PPM as a function of the sampling rate, number of training symbols, and modulation index, in AWGN channel.

where we omit the index $k$ for readibility. The SNR at the output of the equalizer is given by

$$
(S N R)_{\text {out }}=10 \log _{10}\left(\frac{E\left[\left|h_{\mathrm{eq}} h_{\mathrm{f}, \mathrm{c}} s\right|^{2}\right]}{E\left[\left|h_{\mathrm{eq}} n+h_{\mathrm{f}, \mathrm{c}} s n_{\mathrm{h}}+n n_{\mathrm{h}}\right|^{2}\right]}\right)
$$

As the noise affecting the data symbol and the estimated channel are not correlated, the SNR degradation is given by

$$
\begin{aligned}
(S N R)_{\mathrm{deg}} & =10 \log _{10} \frac{E\left[\left|h_{\mathrm{eq}} n+h_{\mathrm{f}, \mathrm{c}} s n_{\mathrm{h}}+n n_{\mathrm{h}}\right|^{2}\right]}{E\left[\left|h_{\mathrm{eq}} n\right|^{2}\right]} \\
& =10 \log _{10}\left(1+\frac{\sigma_{h_{\mathrm{f}, \mathrm{c}}}^{2} \sigma_{\mathrm{s}}^{2}}{N_{\mathrm{pch}} \sigma_{h_{\mathrm{eq}}}^{2}}+\frac{\sigma_{\mathrm{n}}^{2}}{N_{\mathrm{pch}} \sigma_{h_{\mathrm{eq}}}^{2}}\right)
\end{aligned}
$$

where $\sigma_{h_{\mathrm{eq}}}^{2}=E\left[\mathbf{h}_{\mathrm{eq}}^{H} \mathbf{h}_{\mathrm{eq}}\right]$, and $\sigma_{h_{\mathrm{f}, \mathrm{c}}}^{2}=E\left[\mathbf{h}_{\mathrm{f}, \mathrm{c}}^{H} \mathbf{h}_{\mathrm{f}, \mathrm{c}}\right]$. The second term in (26) dominates at low SNR, where $\sigma_{h_{\mathrm{eq}}}^{2} \approx \sigma_{h_{\mathrm{f}, \mathrm{c}}}^{2} \sigma_{\mathrm{s}}^{4} / \sigma_{\mathrm{n}}^{4}$, and may lead to the conclusion that a large number of training symbols is required to achieve robust channel estimation. As shown in the next section, however, an evaluation of the performance degradation in terms of BER shows this is not the case.

2) Degradation in Terms of Bit Error Rate: While the SNR degradation provides an assessment means which is independent of any particular demodulation algorithm, the BER constitutes the ultimate design criterion. Fig. 6 shows the BER curves for PPM modulation in AWGN conditions. When operating in reduced quality mode (low receiver bandwidth, sampling rate, and power consumption), the performance of the receiver is constrained by the limited signal energy being captured and number of available samples, even if the modulation index $\Delta$ is increased. As a result, the preamble length can be kept small (e.g. $N_{\text {pch }}=8$ symbols) without impacting the reception quality in this mode of operation. On the contrary, the number of training symbols affects the BER if the receiver operates in improved quality mode (high sampling rate and power consumption). As expected from the SNR analysis, increasing the preamble length improves the BER with diminishing return. 


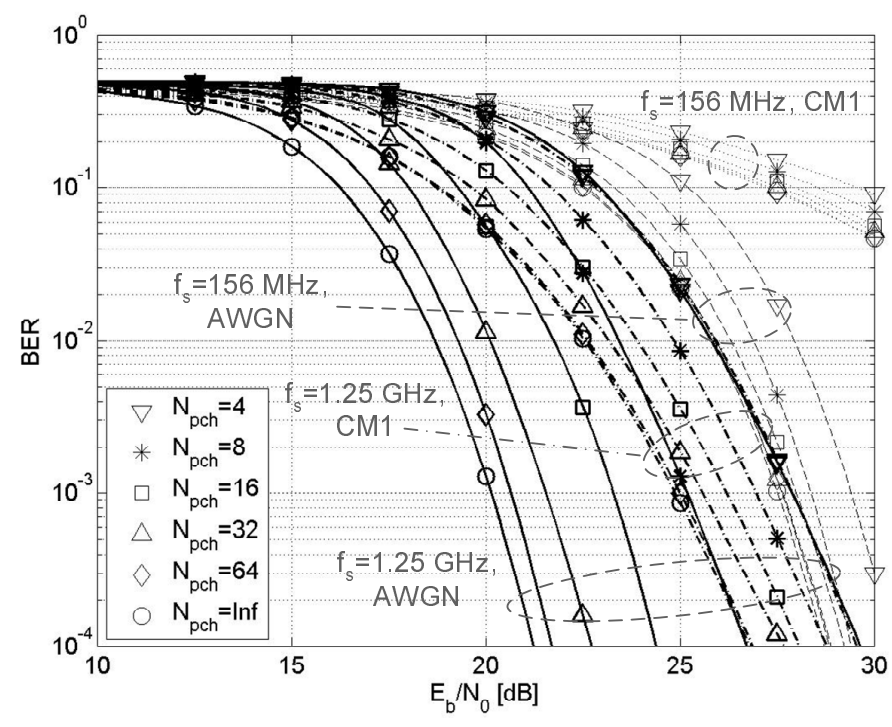

Fig. 7. BER for PAM as a function of the sampling rate and the number of training symbols, in AWGN and multipath channel conditions.

In multipath channel conditions, the receiver becomes less sensitive to the preamble length, as illustrated by Fig. 7 for PAM modulation. The degradation remains minor $(1 \mathrm{~dB})$ if $N_{\text {pch }}=16$. Similar conclusions apply to PPM and other channel models.

As a result, the receiver is robust against imperfect knowledge of the propagation channel, since a limited number of training symbols is sufficient. In addition, the simulation results confirm the flexibility of the receiver with respect to conventional architectures, such as selective or partial Rake receivers. Indeed, these require proper estimation of the tap positions and amplitudes, whereas the training of the simplified MMSE equalizer is a straightforward operation. Finally, these results also suggest flexible channel estimation schemes. For example, the transmitter could adapt the number of training symbols sent depending on the operation mode of the receiver, which can be modified according to the remaining battery level. The receiver and transmitter need to be in a feedback loop in this case.

\section{B. Imperfect Estimation of the Signal-to-Noise Ratio}

In addition to the frequency response of the propagation channel, the suboptimal MMSE equalizer requires the estimation of the signal-to-noise ratio at the input of the equalizer. We consider here the impact of an estimation error in this term, assuming that the propagation channel is perfectly known. In order to assess the sensitivity of the receiver's performance irrespective of any particular SNR estimation algorithm, we introduce an error factor $\alpha$ in (11), i.e. $\mathbf{H}_{\mathrm{eq}}(\alpha)=\mathbf{H}_{\mathrm{f}, \mathrm{c}}^{H}\left(\mathbf{H}_{\mathrm{f}, \mathrm{c}} \mathbf{H}_{\mathrm{f}, \mathrm{c}}^{H}+(\alpha S N R)^{-1} \mathbf{I}_{N_{\mathrm{s}}}\right)^{-1}$. Figure 8 illustrates the relative BER degradation $B E R(\alpha) / B E R(0)$ for various sampling rates and AWGN power levels. The performance degradation remains limited even for an SNR estimation error of $10 \mathrm{~dB}$. The receiver is more sensitive to errors when the BER is low (i.e. at high sampling rate or high $\left.E_{\mathrm{b}} / N_{0}\right)$. However, the likelihood of misestimating significantly the SNR in such conditions is decreasing, so that
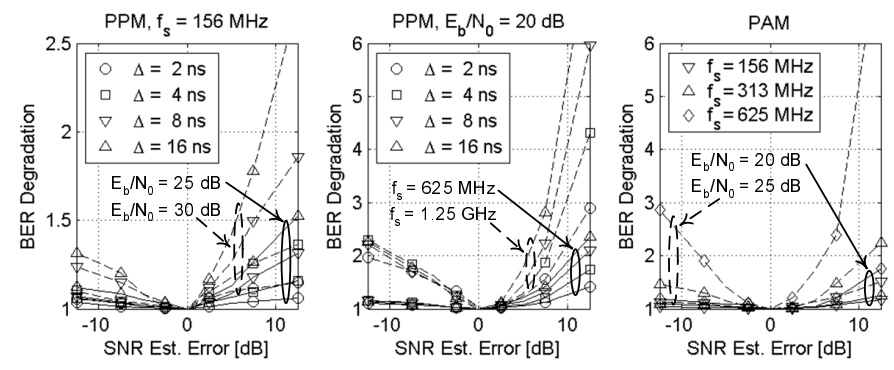

Fig. 8. Sensitivy of the receiver to errors in the estimated SNR in multipath conditions (CM1).

we can safely conclude that the sensitivity of the receiver to SNR errors remains low.

\section{ReCEIVER ARCHITECTURE}

A possible receiver architecture is shown in Fig. 9. The sampling rate is limited by the achievable speed of current ADC technology and power consumption constraints from the system specification. A fraction of the total signal bandwidth is selected by the filters and brought to baseband without aliasing by the mixer in the analog front-end.

As an alternative architecture, the sampling rate and bandwidth of the filters can be fixed at a high value (e.g., $1.25 \mathrm{GHz}$ ) and the mixer frequency remains tunable. The subband within the spectrum window of the RF filters is then selected by filters in the digital domain. Although the ADC continuously works at the highest sampling rate tolerated by implementation concerns and power consumption requirements, this architecture allows for a more flexible selection of the signal band to be processed.

A direct bandpass sampling architecture [17] should be avoided in the case of pulsed UWB signals with a spectrum up to $10.6 \mathrm{GHz}$. Such architecture imposes indeed severe requirements on aperture jitter and noise folding. Assuming a worst case analysis based on the classical aperture jitter noise model $S N R_{\mathrm{aj}}=-20 \log \left(2 \pi f_{\max } \sigma_{T_{\mathrm{aj}}}\right)$, where $f_{\max }=10.6 \mathrm{GHz}$, the RMS value of the aperture jitter $\sigma_{T_{\mathrm{aj}}}$ should be in the order of magnitude of picoseconds for the aperture noise to be negligible with respect to the available SNR. In addition, the wideband noise at the sampling circuitry is aliased to the signal band. This noise folding issue is exacerbated by the extreme subsampling factor $f_{\mathrm{c}} / f_{\mathrm{s}}$. A direct conversion architecture (Fig. 9) is therefore preferable.

As shown in [28], the ADC bit width requirements are moderate (3-4 bits), which guarantees low power consumption. The implementation of a line spectrum estimation method, such as ESPRIT, is the most complex part of the digital backend. It requires expanding a data matrix using a Singular Value Decomposition (SVD), solving a least-squares problem and computing the roots of a polynomial. However, the implementation of ESPRIT is reduced to the SVD in the particular case of $K=1$, i.e. a single pulse per period $T_{\mathrm{f}}$, as the other operations become trivial. The implementability is improved further by constructing a square data matrix $\mathbf{Y}$, i.e. if $m=\left\lceil N_{\mathrm{S}} / 2\right\rceil$. (13) and simulations confirm that the performance degradation with respect to the optimal value of $m$ remains negligible. As a result, the SVD can be efficiently realized with a square 


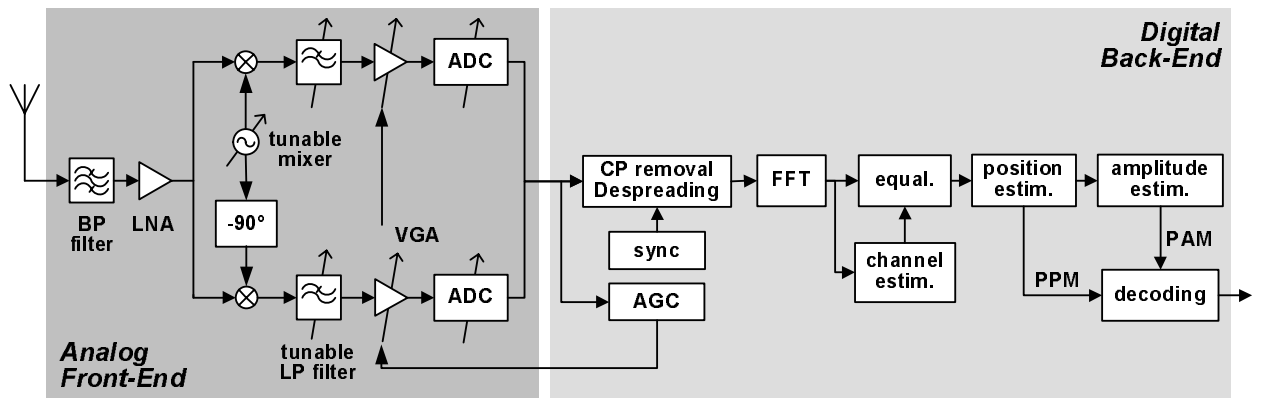

Fig. 9. Subsampling receiver architecture.

systolic array of processors performing the diagonalization task using CORDICs ([29], [30]), without having to carry out a preliminary QR decomposition [31]. Assuming a maximum sampling rate of $f_{\mathrm{s}}=32 / T_{\mathrm{f}}=625 \mathrm{MHz}$, for instance, the SVD requires an array of $8 \times 8$ processors. Such a systolic array can easily accommodate different sampling rates, providing therefore an implementation which can support the flexibility needed at system-level by the algorithms. Indeed, the size of the data matrix is reduced at lower sampling rates, and unnecessary processors can be switched off to minimize the power consumption. Rapid prototyping techniques have allowed us to estimate a receiver complexity of 2 MGates, which is in the same range as the few digital Rake UWB receivers reported [11], [32].

The complexity of the FFT is small compared to that of ESPRIT, by virtue of the limited number of samples. As an example, a 32-point FFT is required in case of a maximum sampling rate of $f_{\mathrm{s}}=32 / T_{\mathrm{f}}=625 \mathrm{MHz}$. Several implementations of 128-point FFTs supporting 1 GSamples/s for UWB applications have been reported, such as [33]-[36], and typically require 100 to 300 KGates at clock speeds ranging from 125 to $500 \mathrm{MHz}$. In addition, the FFT can be implemented on a systolic array of simple processors, such as e.g. [37], like ESPRIT. By doing so, the cost of the FFT is even further minimized if the same architecture is used for both operations.

\section{CONCLUSIONS}

This paper has presented and assessed a flexible subsampling receiver for pulsed UWB communication in the 3.1$10.6 \mathrm{GHz}$ frequency band. The demodulation performance of the receiver can be traded for power consumption and is essentially limited by the amount of energy captured, i.e. the undersampling ratio. The subsampling receiver is robust against imperfections of the equalizer, and has a complexity comparable with classical digital receivers which have been realized and reported in the literature.

\section{REFERENCES}

[1] A. F. Molisch et al., "A low-cost time-hopping impulse radio system for high data rate transmission," EURASIP J. Applied Signal Proc., vol. 2005, no. 1, pp. 397-412, 2005.

[2] R. T. Hoctor and H. W. Tomlinson, "An overview of delay-hopped, transmitted-reference RF communications," in Technical Inform. Series. G.E. Research and Development Center, 2002, pp. 1-29.
[3] Q. Dang, A. Trindade, A.-J. van der Veen, and G. Leus, "Signal model and receiver algorithms for a transmit-reference ultra-wideband communication system," IEEE J. Select. Areas Commun., vol. 24, no. 4, pp. 773-779, 2006.

[4] D. Cassioli, M. Win, F. Vatalaro, and A. Molisch, "Low complexity Rake receivers in ultra-wideband channels," IEEE Trans. Wireless Commun., vol. 6, no. 4, pp. 1265-1275, 2006.

[5] Lottici, V. and D'Andrea, A. and U. Mengali, "Channel estimation for ultra-wideband communications," IEEE J. Select. Areas Commun., vol. 20, no. 9, pp. 1638-1645, 2002.

[6] R. Scholtz, D. Pozar, and W. Namgoong, "Ultra-wideband radio," EURASIP J. Applied Signal Processing, no. 3, pp. 252-272, 2005.

[7] P. Schvan, D. Pollex, S.-C. Wang, C. Falt, and N. Ben-Hamida, "A 22 GS/s 5b ADC in 0.13um SiGe BiCMOS," in Proc. IEEE Int. Solid-State Circuits Conf., 2006, pp. 572-573.

[8] B. Le, T. Rondeau, J. Reed, and C. Bostian, "Analog-to-digital converters," IEEE Signal Processing Mag., vol. 22, no. 6, pp. 69-77, 2005.

[9] L. Smaini and D. Hélal, "RF digital transceiver for impulse radio ultra wide band commun.," in Proc. European Solid-State Circuits Conf. (ESSCIRC) Workshop, 2004.

[10] S. Hoyos, B. Sadler, and G. Arce, "Monobit digital receivers for ultrawideband communications," IEEE Trans. Wireless Commun., vol. 4, no. 4, pp. 1337-1344, July 2005.

[11] R. Blazquez, P. Newaskar, F. Lee, and A. Chandrakasan, "A baseband processor for impulse ultra-wideband communications," IEEE J. SolidState Circuits, vol. 40, no. 9, pp. 1821-1828, 2005.

[12] L. Feng and W. Namgoong, "An oversampled channelized UWB receiver with transmitted reference modulation," IEEE Trans. Wireless Commun., vol. 5, no. 6, pp. 1497-1505, 2006.

[13] S. Hoyos and B. Sadler, "Ultra-wideband analog-to-digital conversion via signal expansion," IEEE Trans. Veh. Technol., vol. 54, no. 5, pp. 1609-1622, 2005.

[14] R. Thirugnanam, D. Ha, and S. Choi, "Design of a 4-bit 1.4 GSamples/s low power folding ADC for DS-CDMA UWBTransceivers," in Proc. IEEE Int. Conf. Ultra-Wideband (ICUWB), 2005, pp. 536-541.

[15] P. Figueiredo et al., "A 90nm CMOS 1.2V 6b 1GS/s two-step subranging ADC," in Proc. IEEE Int. Solid-State Circ. Conf., 2006, pp. 2320-2329.

[16] S. Park, Y. Palaskas, and M. P. Flynn, "A 4-GS/s 4-bit Flash ADC in 0.18- $\mu \mathrm{m}$ CMOS," IEEE J. Solid-State Circuits, vol. 42, no. 9, pp. 1865-1872, 2007.

[17] M. Chen and R. Brodersen, "A subsampling UWB impulse radio architecture utilizing analytic signaling," IEICE Trans. Electron., vol. E88-C, no. 6, pp. 1114-1121, 2005.

[18] M. Vetterli, P. Marziliano, and T. Blu, "Sampling signals w. finite rate of innovation," IEEE Trans. Signal Processing, vol. 50, pp. 1417-1428, 2002.

[19] J. Kusuma, A. Ridolfi, and M. Vetterli, "Sampling of communication systems with bandwidth expansion," in Proc. IEEE Int. Conf. Commun., 2002, pp. 1601-1605.

[20] P. Stoica and R. Moses, Introduction to Spectral Analysis. Prentice Hall, 1997.

[21] J. Zhang, T. Abhayapala, and R. Kennedy, "Principal components tracking algorithms for synchronization and channel identification in UWB systems," in Proc. IEEE $8^{\text {th }}$ Int. Symp. Spread Spectr. Technol. \& Applic., 2004, pp. 369-373.

[22] J. Foerster, "Channel Modeling Sub-Committee Report Final (IEEE P802.15-02/490r1-SG3a)," 2003.

[23] I. Maravić and M. Vetterli, "Low-complexity subspace methods for channel estimation and synchronizationin ultra-wideband systems," in Proc. Int. Workshop UWB Systems, 2003. 
[24] R. Roy and T. Kailath, "ESPRIT-estimation of signal parameters via rotational invariance techniques," IEEE Trans. Acoust., Speech, Signal Proc., vol. 37, no. 7, pp. 984-995, 1989.

[25] P. Stoica and T. Söderström, "Statistical analysis of MUSIC and subspace rotation estimates of sinusoidal frequencies," IEEE Trans. Signal Processing, vol. 39, pp. 1836-1847, 1991.

[26] Y. Hua and T. Sarkar, "Matrix pencil method for estimating parameters of exponentially damped/undamped sinusoids in noise," IEEE Trans. Acoust., Speech, Signal Processing, vol. 38, no. 5, pp. 814-824, 1990.

[27] J.-P. Delmas, "Asymptotic performance of second-order algorithms," IEEE Trans. Signal Processing, vol. 50, no. 1, pp. 49-57, 2002.

[28] Y. Vanderperren, G. Leus, and W. Dehaene, "An approach for sprecifying the ADC and AGC requirements for UWB digital receivers," in Proc. IET Seminar on UWB Syst., Technologies \& Applications, 2006.

[29] J. R. Cavallaro and A. C. Elster, "A CORDIC processor array for the SVD of a complex matrix," in SVD and Signal Processing II (Algorithms, Analysis and Applications). Elsevier, 1991, pp. 227-239.

[30] N. D. Hemkumar, "A systolic VLSI architecture for complex SVD," Master's thesis, Rice University, 1991.

[31] G. H. Golub and C. F. V. Loan, Matrix Computations (3 ${ }^{\text {rd }}$ ed.). Johns Hopkins University Press, 1996.

[32] A. Koyama et al., "A DSSS UWB digital PHY/MAC transceiver for wireless ad hoc mesh networks with distributed control," in Proc. IEEE Int. Solid-State Circuits Conf. (ISSCC), 2006, pp. 992-1001.

[33] Y.-W. Lin, H.-Y. Liu, and C.-Y. Lee, "A 1-GS/s FFT/IFFT processor for UWB applications," IEEE J. Solid-State Circuits, vol. 40, no. 8, pp. 1726-1735, 2005.

[34] L. Liu, J. Ren, X. Wang, and F. Ye, "Design of low-power, 1GS/s throughput FFT processor for MIMO-OFDM UWB communication system," in Proc. IEEE Int. Symp. Circuits \& Systems (ISCAS), 2007, pp. 2594-2597.

[35] H. Lee and M. Shin, "A high-speed low-complexity two-parallel radix$2^{4}$ FFT/IFFT processor for UWB applications," in Proc. IEEE Asian Solid-State Circuits Conf. (ASSCC), 2007, pp. 284-287.

[36] G. Zhang and F. Chen, "Parallel FFT with CORDIC for ultra wide band," in Proc. IEEE Int. Symp. Personal, Indoor Mobile Radio Commun. (PIMRC), F. Chen, ed., vol. 2, 2004, pp. 1173-1177 vol. 2.

[37] J. Nash, "Systolic architecture for computing the discrete fourier transform on FPGAs," in Proc. 13th IEEE Symp. Field-Programmable Custom Comp. Machines (FCCM), 2005, pp. 305-306.

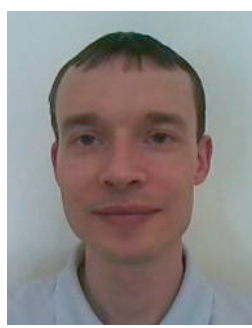

Yves Vanderperren was born in Brussels, Belgium, in 1975. He received the M. Sc. degree in electrical engineering in 1998 from the Université Catholique de Louvain. His thesis focused on multiresolution coding of MPEG4 video streams based on discrete wavelet transform. From 1998 to 2003, he was Design Engineer and System Architect at Alcatel Microelectronics, which was acquired in 2002 by STMicroelectronics. He worked on the feasibility study and the hardware design of high speed ATM and wireless LAN systems. His work on synchronization algorithms for WLAN has been patented (EP1593247, US7424067). In December 2003, he started his Ph.D. at the Katholieke Universiteit Leuven on an ultrawideband receiver based on subsampling techniques. He developed during his industrial and academic experience a cross-disciplinary expertise which associates the domains of algorithmic development, hardware implementation, and design flow aspects. He is program committee member of various conferences on digital architectures and design languages. He is currently an examiner at the European Patent Office in The Hague, The Netherlands, focusing on diagnostic systems in the medical field, in particular signal processing techniques and their implementation.

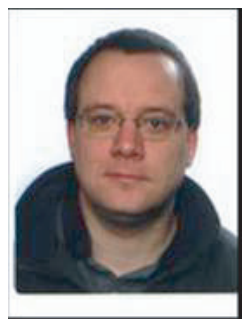

Wim Dehaene was born in Nijmegen, The Netherlands, in 1967. He received the M. Sc. degree in electrical and mechanical engineering in 1991 from the Katholieke Universiteit Leuven. In November 1996 he received the $\mathrm{Ph}$. D degree at the Katholieke Universiteit Leuven. His thesis is entitled CMOS integrated circuits for analog signal processing in hard disk systems.

After receiving the M. Sc. Degree Wim Dehaene was a research assistant at the ESAT-MICAS Laboratory of the Katholieke Universiteit Leuven. His research involved the design of novel CMOS building blocks for hard disk systems. The research was first sponsored by the IWONL (Belgian Institute for Science and Research in Industry and agriculture) and later by the IWT (the Flemish institute for Scientific Research in the Industry). In November 1996 Wim Dehaene joined Alcatel Microelectronics, Belgium. There he was a senior project leader for the feasibility, design and development of mixed mode Systems on Chip. The application domains were telephony, xDSL and high speed wireless LAN. In July 2002 Wim Dehaene joined the staff of the ESAT-MICAS laboratory of the Katholieke Universiteit Leuven where he is now a professor. His research domain is circuit level design of digital circuits. The current focus is on ultra low power signal processing and memories in advanced CMOS technologies. Part of this research is performed in cooperation with IMEC, Belgium where he is also a part time principal scientist. Wim Dehaene is teaching several classes on digital circuit and system design. Wim Dehaene is a senior member of the IEEE.

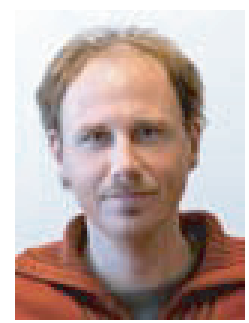

Geert Leus was born in Leuven, Belgium, in 1973. He received the electrical engineering degree and the $\mathrm{PhD}$ degree in applied sciences from the Katholieke Universiteit Leuven, Belgium, in June 1996 and May 2000, respectively. He has been a Research Assistant and a Postdoctoral Fellow of the Fund for Scientific Research - Flanders, Belgium, from October 1996 till September 2003. During that period, Geert Leus was affiliated with the Electrical Engineering Department of the Katholieke Universiteit Leuven, Belgium. Currently, Geert Leus is an Associate Professor at the Faculty of Electrical Engineering, Mathematics and Computer Science of the Delft University of Technology, The Netherlands. During the summer of 1998, he visited Stanford University, and from March 2001 till May 2002 he was a Visiting Researcher and Lecturer at the University of Minnesota. His research interests are in the area of signal processing for communications. Geert Leus received a 2002 IEEE Signal Processing Society Young Author Best Paper Award and a 2005 IEEE Signal Processing Society Best Paper Award. He is the Chair of the IEEE Signal Processing for Communications Technical Committee, and an Associate Editor for the IEEE TRANSACTIONS ON Signal PROCESSING and the EURASIP JoURnAL on ApPlied Signal Processing. In the past, he has served on the Editorial Board of the IEEE Signal Processing LetTERS and the IEEE TRANSACTIONS ON WIRELESS COMMUNICATIONS. 